\title{
ESTRUTURA, COMPOSIÇÃO FLORÍSTICA E CARACTERIZAÇÃO SUCESSIONAL EM REMANESCENTE DE FLORESTA ESTACIONAL SEMIDECIDUAL NO SUDESTE DO BRASIL ${ }^{1}$
}

Laíne Silveira Corrêa ${ }^{2}$, Eliana Cardoso-Leite ${ }^{3}$, Ana Carolina Devides Castello ${ }^{4}$, Samuel Coelho ${ }^{5}$, Alessandra Rocha Kortz ${ }^{6}$, Fernando Nadal Junqueira Villela ${ }^{7}$ e Ingrid Koch ${ }^{8}$

\begin{abstract}
RESUMO - Este estudo foi realizado em remanescente de Floresta Estacional Semidecidual (FES), localizado em Sorocaba (SP, Brasil), com o objetivo de verificar seu estágio sucessional e inferir sobre a composição futura. A hipótese deste artigo previa que esse remanescente apresentaria regeneração com baixa diversidade de espécies, predomínio de espécies pioneiras de dossel, ausência de espécies residentes no sub-bosque e predomínio de espécies anemocóricas e autocóricas. Foi utilizado o método de parcelas, considerando os indivíduos em três classes de tamanho, duas delas referentes ao sub-bosque e uma ao dossel $(1-\leq 100 \mathrm{~cm}$; 2 - >100 cm e circunferência à altura do solo $\leq 15 \mathrm{~cm} ; 3$ - CAP $\geq 15 \mathrm{~cm}$ ). Foram amostradas 146 espécies, sendo os maiores valores de diversidade encontrados no dossel e nas espécies de sub-bosque da classe 2. A similaridade entre as três classes foi baixa. Das espécies com maior valor de importância, cinco são pioneiras e cinco são não pioneiras, e as espécies com maiores valores de regeneração natural são consideradas como não pioneiras e residentes. Contrariando a hipótese inicial, essas características, somadas ao predomínio de espécies zoocóricas nas três classes, indicam que o remanescente está em estágio intermediário de sucessão e possui condições microclimáticas para a entrada e permanência de espécies que necessitam de áreas mais sombreadas. Espécies como Aspidosperma olivaceum, Cordia trichotoma, Ocotea elegans e Guarea guidonia, consideradas não pioneiras e transitórias, foram amostradas somente nas classes 1 e 2, indicando a futura substituição de espécies e o avanço sucessional do remanescente.
\end{abstract}

Palavras-chave: Regeneração natural; Sucessão secundária; Sub-bosque.

\section{STRUCTURE, FLORISTIC COMPOSITION AND SUCCESSIONAL CHARACTERIZATION OF FRAGMENTS OF SEMIDECIDUOUS SEASONAL FOREST IN SOUTHEAST BRAZIL}

\begin{abstract}
This study was conducted in a fragment of semideciduous seasonal forest (SSF), located in Sorocaba (SP, Brazil), in order to verify its successional stage and infer from the future composition. Our hypothesis was that the fragment would present regeneration with low diversity of species; with predominance of pioneer species of canopy and absence of resident species in the understory and predominance of anemochoric and autochoric species. We used the parcels method, considering the individuals in three size classes, two of them referring to rhe understory and one to the canopy $(1-\leq 100 \mathrm{~cm} ; 2->100 \mathrm{~cm}$ and circumference at soil height $\leq 15 \mathrm{~cm} ; 3-\geq 15 \mathrm{~cm}$. We sampled 146 species, and class 2 understory species presented the highest diversity levels. The similarity between the three classes was low. Considering the species with the highest importance values, five are pioneer and five are non pioneer. Contesting the initial hypothesis, these characteristics, plus the predominance of the zoochorich species in the three species, indicate that the fragment is in an intermediate stage of succession and has microclimatic conditions that allow the entrance and presence
\end{abstract}

\footnotetext{
${ }^{1}$ Recebido em 30.08.2013 aceito para publicação em 24.07.2014.

${ }^{2}$ Programa de Pós Graduação em Biologia Vegetal, Universidade Estadual de Campinas, Brasil. E-mail: <laine06correa@gmail.com>. ${ }^{3}$ Departamento de Ciências Ambientais, Universidade Federal de São Carlos, Campus de Sorocaba, Brasil. E-mail: <eliana.leite@ufscar.br>.

${ }^{4}$ Programa de Pós Graduação em Ciências Biológicas (Botânica), Universidade Estadual Paulista Júlio de Mesquita Filho, Brasil. E-mail: <carol.dcastello@gmail.com>.

${ }^{5}$ Programa de Pós Graduação em Sustentabilidade na Gestão Ambiental, Universidade Federal de São Carlos, Campus de Sorocaba, Brasil. E-mail: <samucabeca@hotmail.com>

${ }^{6}$ Programa de Pós Graduação Centre for Biological Diversity, University of St Andrews, Escócia. E-mail: <ark4@st-andrews.ac.uk>.

${ }^{7}$ Departamento de Geografia, Faculdade de Filosofia Letras e Ciências Humanas, Universidade de São Paulo, Brasil. E-mail: <fernando.villela@ufscar.br>.

${ }^{8}$ Departamento de Biologia, Universidade Federal de São Carlos, Campus Sorocaba, Brasil. E-mail: <ingrid@ufscar.br>
} 
of species that need areas with more shadow. Species like Aspidosperma olivaceum, Cordia trichotoma, Ocotea elegans and Guarea guidonia, considered as non pioneer and transitory species, were sampled only in classes 1 and 2, which shows the future species replacement and the sucessional progress of the fragment.

Keywords: Natural regeneration; Secondary sucession; Understory.

\section{INTRODUÇÃO}

A Mata Atlântica está entre os ecossistemas tropicais mais ameaçados do mundo, apresentando atualmente entre $11,4 \%$ e $16 \%$ de sua cobertura original (RIBEIRO et al., 2009). A Floresta Estacional Semidecidual (FES) é o tipo florestal do domínio Mata Atlântica mais intensamente destruído ao longo dos últimos séculos, devido à fertilidade do solo e ao relevo favorável à agricultura e pecuária, bem como à intensiva modernização da atividade agrícola em anos recentes (LOPES et al., 2012). Atualmente, a maioria dos remanescentes de FES é constituída de florestas secundárias com tamanhos reduzidos, diferentes idades e diversos históricos de perturbação (KRONKA et al., 2005). Assim, cada um desses remanescentes de vegetação possui uma flora particular (LIEBSCH et al., 2008), própria dessa formação florestal (CARDOSO-LEITE; RODRIGUES, 2008).

O estado de conservação dos remanescentes florestais tropicais pode ser verificado por sua estrutura vertical. A análise da riqueza de espécies do sub-bosque possibilita inferir sobre o potencial de regeneração das espécies existentes no dossel, nos diferentes grupos ecológicos, assim como estimar se a dinâmica de substituição de espécies é mantida em diferentes momentos no decorrer da sucessão (SWAINE; WHITMORE, 1988). Adicionalmente, a proporção entre as síndromes de dispersão pode indicar se os processos ecológicos entre plantas e animais estão sendo mantidos (AIZEN; FEINSINGER, 1994).

Áreas com histórico de intensivo uso agropastoril, bem como outras atividades antrópicas, apresentam, em geral, baixa disponibilidade de nutrientes e baixa densidade ou inexistência do banco de sementes e plântulas, o que resulta em processos de regeneração com limitada diversidade de espécies (ENGEL; PARROTA, 2008). Considerando um pequeno remanescente de FES situado em uma matriz mista de uso agropastoril, com idade aproximada de 50 anos, objetivou-se neste estudo inferir sobre seu estágio sucessional e composição futura, a fim de contribuir para o entendimento da regeneração natural nesse tipo de formação vegetal. A hipótese inicial previa que esse remanescente apresentaria regeneração com baixa diversidade de espécies, predomínio de espécies pioneiras de dossel, ausência de espécies residentes no sub-bosque e predomínio de espécies anemocóricas e autocóricas.

\section{MATERIAL E MÉTODOS}

\section{1. Área de estudo}

O remanescente de vegetação localiza-se na região Sudoeste do Município de Sorocaba (2334'57" S; 2335'25" S e 4731'50" W; 47³1'00" W), com área total de 27,3 hectares e predomínio de Floresta Estacional Semidecidual (IBGE, 2012) com elementos de Cerrado. Este está inserido em uma região de transição entre o Planalto Atlântico e a Depressão Periférica Paulista, com solos dos tipos Argissolo Vermelho-Amarelo distrófico e Latossolo Vermelho distrófico (OLIVEIRA et al., 1999). O clima da região é classificado como Cwa (KÖEPPEN, 1948), quente e chuvoso, com inverno seco.

Fotografias aéreas da década de 1960 (Figura 1) demonstram que a área estudada está em processo de regeneração, pois o remanescente era menor e a vegetação bastante esparsa em alguns trechos, em matriz com áreas de pastagem e de plantio de Eucalyptus spp. As parcelas foram alocadas em sete pontos dentro da área Campus de Sorocaba, da Universidade Federal de São Carlos (Figura 1).

\subsection{Amostragem}

Para a amostragem, foi utilizado o método de parcelas (MÜELLER-DOMBOIS; ELLENBERG, 1974), sendo alocadas quatro nos pontos 1, 2, 3 e 4 e uma nos pontos 5, 6 e 7, totalizando 19 parcelas. Os indivíduos foram considerados em três classes de tamanho: classe 1 - indivíduos com no mínimo dois pares de folhas (FENNER, 1987) e altura máxima de 100 cm; classe 2 - indivíduos com altura superior a $100 \mathrm{~cm}$ e circunferência à altura do solo $<15 \mathrm{~cm}$; e classe 3 - indivíduos com circunferência à altura do peito $\geq 15 \mathrm{~cm}$. As classes 


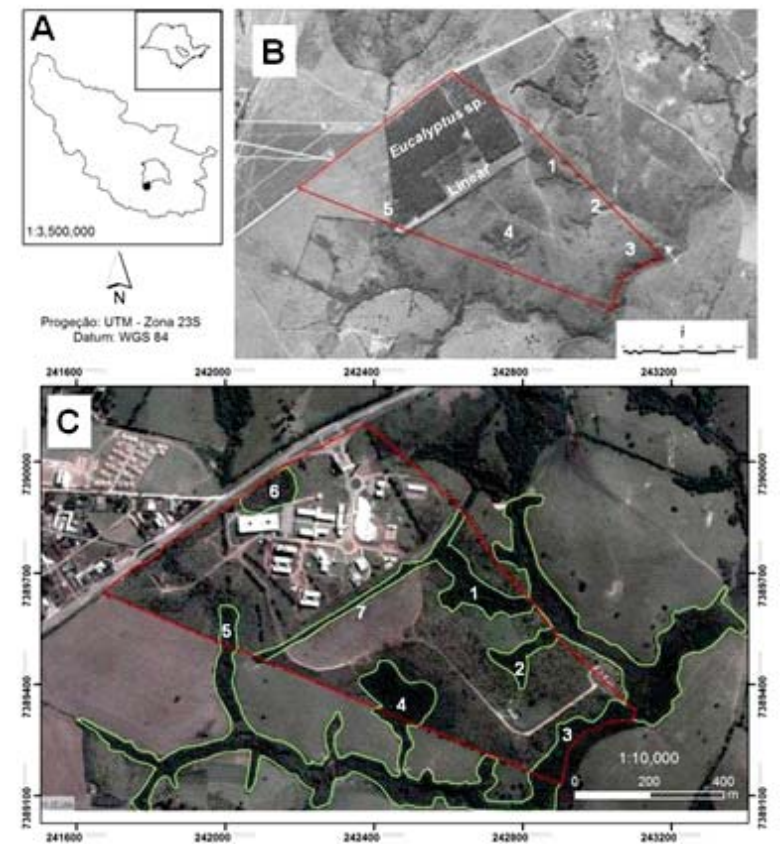

Figura 1 - Fotografia aérea do fragmento de FES em Sorocaba (perímetro do Campus da UFSCar em vermelho): A) Localização da área de estudo; B) Foto área da área de estudo em 1962 (Base 1962); e C) Foto aérea da área de estudo em 2006 (Aerocarta 2006).

Figure 1-Aerial photograph of the fragment of SSF in Sorocaba (the campus perimeter of UFSCar is highlighted in red): A) Localization of the study area. B) Aerial photograph of the study area in 1962 (Base 1962); and C) Aerial photograph of the study area in 2006 (Aerocarta, 2006).

de tamanho foram amostradas em parcelas de: $10 \times 10$ m para a classe $3\left(1.900 \mathrm{~m}^{2}\right)$; 5 x $5 \mathrm{~m}$ para a classe 2 , no interior das parcelas maiores $\left(475 \mathrm{~m}^{2}\right) ; 1 \mathrm{x} 1 \mathrm{~m}$ na classe 1 , no centro das parcelas maiores $\left(19 \mathrm{~m}^{2}\right)$. As classes 1 e 2 referem-se ao sub-bosque e a classe 3, ao dossel.

O material coletado foi prensado, seco em estufa e doado para a coleção do Herbário da Universidade Federal de São Carlos - Campus Sorocaba. As identificações foram feitas a partir de literatura pertinente, comparação com materiais de coleções e auxílio de especialistas. Os indivíduos não identificados foram considerados morfoespécies. O nome das famílias está de acordo com a proposta do Angiosperm Phylogeny Group III (APG III, 2009). As grafias dos nomes das espécies e dos autores foram conferidas em bibliografia pertinente e, ou, à base de dados da Lista de Espécies da Flora do Brasil (2013).

\subsection{Análises}

A estrutura e composição do remanescente foram analisadas, considerando-se as três classes amostradas. Para as classes 1 e 2, calculou-se a Regeneração Natural (RN) das espécies, utilizando valores de frequência e densidade relativas, de acordo com a equação de Volpato (1994): RNij = $($ RDij + RFij $) / 2$, em que RNij é a regeneração natural estimada da i-ésima espécie da j-ésima categoria, em porcentagem; RDij é a densidade relativa da i-ésima espécie da j-ésima categoria; e RFij é a frequência relativa da i-ésima espécie da j-ésima categoria. Para obtenção da Regeneração Natural Total (RNT), realizou-se a soma dos valores de RN de todas as espécies das classes amostradas, obtendo a RNT para cada espécie $(\mathrm{RNTi}=$ “ NRij). Na classe 3, calculou-se o Valor de Importância (VI) (densidade, dominância e frequência relativas somadas), utilizando o software Fitopac 2.1 (SHEPHERD, 2009).

Para caracterização do estágio sucessional, as três classes amostradas foram consideradas conjuntamente com as espécies identificadas nas seguintes categorias: (1) grupo sucessional: pioneiras e não pioneiras (SWAINE; WHITMORE, 1988); (2) síndrome de dispersão: zoocoria, anemocoria e autocoria (LIEBSCH et al., 2008); (3) posição vertical: espécies que completam o ciclo de vida no sub-bosque, consideradas residentes e indivíduos jovens de espécies arbóreas de dossel, consideradas transitórias (GILLIAM et al., 1994); e (4) riqueza (LIEBSCH et al., 2008).

Para comparar a riqueza estimada nas três classes, utilizou-se a análise de rarefação, considerando o número máximo de indivíduos encontrados (345 indivíduos). A similaridade entre as classes foi calculada por meio dos índices: Jaccard, Sørensen e Morisita-Horn (MAGURRAN, 2004; CHAO, 2005), utilizando-se o software EstimateS (CHAO, 2005; COLWELL, 2014). A análise $\hat{\mathrm{a}}_{\mathrm{RC}}$ foi realizada para testar se a diferença entre as classes é significativamente diferente do que o esperado pela criada por modelos nulos (CHASE et al., 2011), realizado por meio do pacote Vegan do software $\mathrm{R}$ (OKSANEN et al., 2014; R CORE TEAM, 2014).

\section{RESULTADOS}

No total foram amostradas 145 espécies e 807 indivíduos (Tabela 1), sem considerar os indivíduos mortos (22 indivíduos). Dessas, sete foram identificadas até gênero, três até família e 23 (15,7\%) como morfoespécies (Tabela 2).

Revista Árvore, Viçosa-MG, v.38, n.5, p.799-809, 2014 
Tabela 1 - Lista de espécies amostradas por classe (C) de tamanho no remanescente de Floresta Estacional Semidecidual em Sorocaba, SP (N: número de indivíduos; GS: grupo sucessional; P: pioneira; NP: não pioneira; SD: síndrome de dispersão; ZOO: zoocoria; ANE: anemocoria; AUT: autocoria; NC: não caracterizada; RN: regeneração natural; e VI: valor de importância.

Table 1 - List of species sampled according to the size class $(C)$ in the Semideciduous Seasonal Forest fragment in Sorocaba, SP. N: number of individuals; SG: successional group; P: pioneer; NP: non pioneer; DS: dispersion syndrome; ZOO: zoochory; ANE: anemochory; AUT: autocory; NC: not classified; NR: Natural Regeneration; and IV: importance value.

\begin{tabular}{|c|c|c|c|c|c|c|c|c|c|c|}
\hline Família & Espécie & $\mathbf{N}$ & C 1 & C 2 & C 3 & GS & SD & $\mathbf{R N}$ & VI & $\mathbf{P V}$ \\
\hline \multirow[t]{2}{*}{ Anacardiaceae } & Lithrea molleoides (Vell.) Engl. & 26 & $\mathrm{X}$ & $\mathrm{X}$ & $\mathrm{X}$ & $\mathrm{P}$ & $\mathrm{ZOO}$ & 3,67 & 22,76 & $\mathrm{~T}$ \\
\hline & Tapirira guianensis Aubl. & 8 & & & $\mathrm{X}$ & NP & $\mathrm{ZOO}$ & - & 7,65 & $\mathrm{~T}$ \\
\hline \multirow[t]{3}{*}{ Annonaceae } & Guatteria nigrescens Mart. & 3 & & & $\mathrm{X}$ & NP & $\mathrm{ZOO}$ & - & 3,19 & $\mathrm{~T}$ \\
\hline & $\begin{array}{l}\text { Rollinia sylvatica (A. St.-Hil.) } \\
\text { Martius }\end{array}$ & 3 & & $\mathrm{X}$ & $\mathrm{X}$ & NP & $\mathrm{ZOO}$ & 0,8 & 2,09 & $\mathrm{~T}$ \\
\hline & Annona dioica A. St.-Hil. & 1 & $\mathrm{X}$ & & & NP & $\mathrm{ZOO}$ & 0,2 & & $\mathrm{R}$ \\
\hline \multirow[t]{2}{*}{ Apocynaceae } & $\begin{array}{l}\text { Aspidosperma olivaceum } \\
\text { Müll. Arg. }\end{array}$ & 1 & & $\mathrm{X}$ & & $\mathrm{NP}$ & ANE & 0,48 & & $\mathrm{~T}$ \\
\hline & $\begin{array}{l}\text { Tabernaemontana catharinensis } \\
\text { A. DC. }\end{array}$ & 3 & $\mathrm{X}$ & $\mathrm{X}$ & $\mathrm{X}$ & $\mathrm{P}$ & $\mathrm{ZOO}$ & 1,29 & 0,96 & $\mathrm{~T}$ \\
\hline Araliaceae & $\begin{array}{l}\text { Dendropanax cuneatus (DC.) } \\
\text { Decne. \& Planch. }\end{array}$ & 14 & $\mathrm{X}$ & $\mathrm{X}$ & & $\mathrm{P}$ & $\mathrm{ZOO}$ & 0,77 & & $\mathrm{R}$ \\
\hline Arecaceae & $\begin{array}{l}\text { Syagrus romanzoffiana (Cham.) } \\
\text { Glassman }\end{array}$ & 4 & & & $\mathrm{X}$ & $\mathrm{NP}$ & $\mathrm{ZOO}$ & - & 6,04 & $\mathrm{~T}$ \\
\hline \multirow[t]{2}{*}{ Asteraceae } & $\begin{array}{l}\text { Dasyphyllum brasiliense } \\
\text { (Spreng.) Cabrera }\end{array}$ & 1 & $\mathrm{X}$ & & & $\mathrm{P}$ & ANE & 0,82 & & $\mathrm{R}$ \\
\hline & $\begin{array}{l}\text { Gochnatia polymorpha } \\
\text { (Less.) Cabrera }\end{array}$ & 22 & $\mathrm{X}$ & $\mathrm{X}$ & $\mathrm{X}$ & $\mathrm{P}$ & ANE & 3,33 & 16,5 & $\mathrm{~T}$ \\
\hline Bignoniaceae & $\begin{array}{l}\text { Handroanthus chrysotrichus } \\
\text { (Mart. ex A. DC.) Mattos }\end{array}$ & 5 & & & $\mathrm{X}$ & $\mathrm{NP}$ & ANE & - & 5,18 & $\mathrm{~T}$ \\
\hline Boraginaceae & $\begin{array}{l}\text { Cordia trichotoma (Vell.) } \\
\text { Arráb. ex Steud. }\end{array}$ & 1 & & $\mathrm{X}$ & & $\mathrm{NP}$ & ANE & 0,48 & & $\mathrm{~T}$ \\
\hline \multirow[t]{2}{*}{ Burseraceae } & $\begin{array}{l}\text { Protium heptaphyllum } \\
\text { (Aubl.) Marchand }\end{array}$ & 32 & & $\mathrm{X}$ & $\mathrm{X}$ & NP & $\mathrm{ZOO}$ & 0,95 & 14,2 & $\mathrm{~T}$ \\
\hline & Protium kleinii Cuatrec. & 3 & $\mathrm{X}$ & & $\mathrm{X}$ & $\mathrm{NP}$ & $\mathrm{ZOO}$ & 0,82 & 2,13 & $\mathrm{~T}$ \\
\hline Cannabaceae & Celtis iguanaea (Jacq.) Sarg. & 5 & & $\mathrm{X}$ & $\mathrm{X}$ & $\mathrm{NP}$ & $\mathrm{ZOO}$ & 0,95 & 3,34 & $\mathrm{~T}$ \\
\hline Caryocaraceae & Caryocar brasiliense Cambess. & 1 & & & $\mathrm{X}$ & NP & $\mathrm{ZOO}$ & & 0,98 & $\mathrm{~T}$ \\
\hline \multirow[t]{2}{*}{ Celastraceae } & Maytenus alaternoides Reissek & 5 & $\mathrm{X}$ & & $\mathrm{X}$ & $\mathrm{P}$ & $\mathrm{ZOO}$ & 1,63 & 2,97 & $\mathrm{~T}$ \\
\hline & $\begin{array}{l}\text { Maytenus ilicifolia Mart. } \\
\text { ex Reissek }\end{array}$ & 7 & & $\mathrm{X}$ & & NP & $\mathrm{ZOO}$ & 3,3 & & $\mathrm{R}$ \\
\hline Clethraceae & Clethra sp. & 1 & $\mathrm{X}$ & & & NC & NC & 0,82 & & NC \\
\hline Ebenaceae & Diospyros inconstans Jacq. & 1 & & $\mathrm{X}$ & & $\mathrm{NP}$ & $\mathrm{ZOO}$ & 0,48 & & $\mathrm{~T}$ \\
\hline Erythroxylaceae & Erythroxylum daphnites Mart. & 2 & & $\mathrm{X}$ & $\mathrm{X}$ & $\mathrm{P}$ & $\mathrm{ZOO}$ & 0,48 & 0,93 & $\mathrm{R}$ \\
\hline \multirow[t]{6}{*}{ Euphorbiaceae } & $\begin{array}{l}\text { Actinostemon concepcionis } \\
\text { (Didr.) Pax }\end{array}$ & 38 & $\mathrm{X}$ & $\mathrm{X}$ & & $\mathrm{NP}$ & AUT & 10,1 & & $\mathrm{R}$ \\
\hline & $\begin{array}{l}\text { Sapium glandulosum } \\
\text { (L.) Morong }\end{array}$ & 1 & & $\mathrm{X}$ & & $\mathrm{P}$ & $\mathrm{ZOO}$ & 0,48 & & $\mathrm{~T}$ \\
\hline & $\begin{array}{l}\text { Sebastiania commersoniana } \\
\text { (Baill.) L.B. Sm. \& Downs }\end{array}$ & 6 & & $\mathrm{X}$ & $\mathrm{X}$ & NP & AUT & 0,71 & 2,34 & $\mathrm{R}$ \\
\hline & $\begin{array}{l}\text { Sebastiania serrata (Baill. } \\
\text { ex Müll. Arg.) Müll. Arg. }\end{array}$ & 2 & $\mathrm{X}$ & & $\mathrm{X}$ & NP & AUT & 0,82 & 0,99 & $\mathrm{~T}$ \\
\hline & Sebastiania sp. & 2 & $\mathrm{X}$ & & & NC & NC & 1,12 & & NC \\
\hline & Euphorbiaceae sp1 & 1 & $\mathrm{X}$ & & & NC & NC & 0,82 & & NC \\
\hline
\end{tabular}


Tabela 1 - Cont.

Table 1 - Cont.

\begin{tabular}{|c|c|c|c|c|c|c|c|c|c|c|}
\hline \multirow[t]{18}{*}{ Fabaceae } & Leptolobium elegans Vogel & 2 & & & $\mathrm{X}$ & NP & ANE & - & 1,38 & $\mathrm{~T}$ \\
\hline & $\begin{array}{l}\text { Albizia niopoides (Spruce } \\
\text { ex Benth.) Burkart }\end{array}$ & 1 & & & $\mathrm{X}$ & NP & AUT & - & 0,98 & $\mathrm{~T}$ \\
\hline & Andira fraxinifolia Benth. & 7 & & & $\mathrm{X}$ & NP & $\mathrm{ZOO}$ & - & 1,95 & $\mathrm{~T}$ \\
\hline & Bauhinia forficata Link & 17 & $\mathrm{X}$ & & $\mathrm{X}$ & $\mathrm{P}$ & AUT & 3,67 & 7,56 & $\mathrm{~T}$ \\
\hline & $\begin{array}{l}\text { Bauhinia longifolia (Bong.) } \\
\text { Steud. }\end{array}$ & 3 & & & $\mathrm{X}$ & $\mathrm{P}$ & AUT & - & 4,15 & $\mathrm{~T}$ \\
\hline & Calliandra foliolosa Benth. & 2 & $\mathrm{X}$ & $\mathrm{X}$ & & NP & AUT & 1,29 & & $\mathrm{R}$ \\
\hline & Copaifera langsdorffii Desf. & 26 & $\mathrm{X}$ & $\mathrm{X}$ & $\mathrm{X}$ & NP & $\mathrm{ZOO}$ & 1,45 & 18,57 & $\mathrm{~T}$ \\
\hline & $\begin{array}{l}\text { Enterolobium contortisiliquum } \\
\text { (Vell.) Morong }\end{array}$ & 18 & & $\mathrm{X}$ & $\mathrm{X}$ & $\mathrm{P}$ & AUT & 1,51 & 2,07 & $\mathrm{~T}$ \\
\hline & Inga marginata Willd. & 1 & & & $\mathrm{X}$ & NP & $\mathrm{ZOO}$ & - & 1,9 & $\mathrm{~T}$ \\
\hline & Inga vera Willd. & 1 & & & $\mathrm{X}$ & $\mathrm{P}$ & $\mathrm{ZOO}$ & - & 2,34 & $\mathrm{~T}$ \\
\hline & $\begin{array}{l}\text { Leucochloron incuriale } \\
\text { (Vell.) Barneby \& J.W. Grimes }\end{array}$ & 7 & $\mathrm{X}$ & & $\mathrm{X}$ & $\mathrm{P}$ & AUT & 1,94 & 1,98 & $\mathrm{~T}$ \\
\hline & $\begin{array}{l}\text { Lonchocarpus } \\
\text { muehlbergianus Hassl. }\end{array}$ & 1 & & $\mathrm{X}$ & & $\mathrm{P}$ & AUT & 0,48 & & $\mathrm{~T}$ \\
\hline & $\begin{array}{l}\text { Lonchocarpus cultratus } \\
\text { (Vell.) A.M.G. Azevedo } \\
\text { \& H.C. Lima }\end{array}$ & 2 & & & $\mathrm{X}$ & NP & ANE & & 1,51 & $\mathrm{~T}$ \\
\hline & $\begin{array}{l}\text { Machaerium paraguariense } \\
\text { Hassl. }\end{array}$ & 1 & & & $\mathrm{X}$ & NP & ANE & - & 1,36 & $\mathrm{~T}$ \\
\hline & Machaerium vestitum Vogel & 2 & & & $\mathrm{X}$ & NP & ANE & & 2,08 & $\mathrm{~T}$ \\
\hline & Machaerium villosum Vogel & 1 & & & $\mathrm{X}$ & NP & ANE & - & 1,21 & $\mathrm{~T}$ \\
\hline & $\begin{array}{l}\text { Piptadenia gonoacantha } \\
\text { (Mart.) J.F. Macbr. }\end{array}$ & 10 & & $\mathrm{X}$ & $\mathrm{X}$ & $\mathrm{P}$ & AUT & 1,65 & 6,29 & $\mathrm{~T}$ \\
\hline & Platypodium elegans Vogel & 1 & & & $\mathrm{X}$ & NP & ANE & - & 1,49 & $\mathrm{~T}$ \\
\hline Lacistemataceae & $\begin{array}{l}\text { Lacistema hasslerianum } \\
\text { Chodat }\end{array}$ & 1 & $\mathrm{X}$ & & & $\mathrm{P}$ & $\mathrm{ZOO}$ & 0,82 & & $\mathrm{R}$ \\
\hline Lamiaceae & Vitex polygama Cham. & 4 & & & $\mathrm{X}$ & NP & $\mathrm{ZOO}$ & - & 3,75 & $\mathrm{~T}$ \\
\hline \multirow[t]{8}{*}{ Lauraceae } & $\begin{array}{l}\text { Cryptocarya moschata } \\
\text { Nees \& Mart. }\end{array}$ & 21 & & & $\mathrm{X}$ & NP & $\mathrm{ZOO}$ & - & 17,09 & $\mathrm{~T}$ \\
\hline & $\begin{array}{l}\text { Endlicheria paniculata } \\
\text { (Spreng.) J.F. Macbr. }\end{array}$ & 14 & $\mathrm{X}$ & $\mathrm{X}$ & $\mathrm{X}$ & NP & $\mathrm{ZOO}$ & 1,63 & 1,01 & $\mathrm{~T}$ \\
\hline & $\begin{array}{l}\text { Nectandra lanceolata } \\
\text { Nees \& Mart. }\end{array}$ & 3 & $\mathrm{X}$ & & $\mathrm{X}$ & NP & $\mathrm{ZOO}$ & - & 5,25 & $\mathrm{~T}$ \\
\hline & $\begin{array}{l}\text { Nectandra megapotamica } \\
\text { (Spreng.) Mez }\end{array}$ & 15 & $\mathrm{X}$ & $\mathrm{X}$ & $\mathrm{X}$ & NP & $\mathrm{ZOO}$ & 4,75 & 2,09 & $\mathrm{~T}$ \\
\hline & $\begin{array}{l}\text { Nectandra reticulata } \\
\text { (Ruiz \& Pav.) Mez }\end{array}$ & 1 & & & $\mathrm{X}$ & NP & $\mathrm{ZOO}$ & - & 1,06 & $\mathrm{~T}$ \\
\hline & Ocotea elegans Mez & 3 & & $\mathrm{X}$ & & NP & $\mathrm{ZOO}$ & 0,7 & & $\mathrm{~T}$ \\
\hline & $\begin{array}{l}\text { Ocotea velutina (Nees) } \\
\text { Rohwer }\end{array}$ & 3 & & & $\mathrm{X}$ & NP & $\mathrm{ZOO}$ & - & 2,24 & $\mathrm{~T}$ \\
\hline & Persea pyrifolia Nees & 11 & & & $\mathrm{X}$ & NP & $\mathrm{ZOO}$ & - & 14,36 & $\mathrm{~T}$ \\
\hline \multirow[t]{2}{*}{ Loganiaceae } & Strychnos sp. & 1 & & $\mathrm{X}$ & & NC & NC & 0,48 & & NC \\
\hline & $\begin{array}{l}\text { Malpighiaceae Byrsonima } \\
\text { intermedia A. Juss. }\end{array}$ & 15 & $\mathrm{X}$ & $\mathrm{X}$ & & NP & $\mathrm{ZOO}$ & 6,28 & & $\mathrm{R}$ \\
\hline \multirow[t]{2}{*}{ Malvaceae } & Guazuma ulmifolia Lam. & 2 & & & $\mathrm{X}$ & $P$ & $\mathrm{ZOO}$ & - & 2,93 & $\mathrm{~T}$ \\
\hline & Luehea candicans Mart. & 1 & $\mathrm{X}$ & & & $\mathrm{P}$ & ANE & 0,8 & & $\mathrm{~T}$ \\
\hline \multirow[t]{3}{*}{ Meliaceae } & $\begin{array}{l}\text { Cabralea canjerana } \\
\text { (Vell.) Mart. }\end{array}$ & 3 & & $\mathrm{X}$ & $\mathrm{X}$ & NP & $\mathrm{ZOO}$ & 0,48 & 3,29 & $\mathrm{~T}$ \\
\hline & $\begin{array}{l}\text { Guarea guidonia } \\
\text { (L.) Sleumer }\end{array}$ & 1 & & $\mathrm{X}$ & & NP & $\mathrm{ZOO}$ & 0,48 & & $\mathrm{~T}$ \\
\hline & Guarea kunthiana A. Juss. & 11 & $\mathrm{X}$ & $\mathrm{X}$ & $\mathrm{X}$ & NP & $\mathrm{ZOO}$ & 1,88 & 0,97 & $\mathrm{~T}$ \\
\hline
\end{tabular}


Tabela 1 - Cont.

Table 1 - Cont

\begin{tabular}{|c|c|c|c|c|c|c|c|c|c|c|}
\hline & Guarea macrophylla Vahl & 2 & & $\mathrm{X}$ & $\mathrm{X}$ & NP & $\mathrm{ZOO}$ & & 1,37 & $\mathrm{~T}$ \\
\hline & Trichilia catigua A. Juss. & 1 & & & $\mathrm{X}$ & $\mathrm{NP}$ & $\mathrm{ZOO}$ & 0,48 & & $\mathrm{R}$ \\
\hline & Trichilia elegans A. Juss. & 26 & $\mathrm{X}$ & $\mathrm{X}$ & $\mathrm{X}$ & NP & $\mathrm{ZOO}$ & 2,73 & 4,6 & $\mathrm{R}$ \\
\hline & Trichilia pallida Sw. & 17 & $\mathrm{X}$ & $\mathrm{X}$ & $\mathrm{X}$ & NP & $\mathrm{ZOO}$ & 2,07 & 3,45 & $\mathrm{R}$ \\
\hline \multirow[t]{2}{*}{ Monimiaceae } & Mollinedia clavigera Tul. & 39 & $\mathrm{X}$ & $\mathrm{X}$ & & NP & $\mathrm{ZOO}$ & 7,74 & & $\mathrm{R}$ \\
\hline & $\begin{array}{l}\text { Mollinedia widgrenii } \\
\text { A. DC. }\end{array}$ & 2 & $\mathrm{X}$ & & & NP & $\mathrm{ZOO}$ & 1,63 & & $\mathrm{R}$ \\
\hline \multirow[t]{5}{*}{ Moraceae } & $\begin{array}{l}\text { Brosimum gaudichaudii } \\
\text { Trécul }\end{array}$ & 3 & & $\mathrm{X}$ & & $\mathrm{P}$ & $\mathrm{ZOO}$ & 0,71 & & $\mathrm{~T}$ \\
\hline & Brosimum glaziovii Taub. & 1 & & & $\mathrm{X}$ & $\mathrm{P}$ & $\mathrm{ZOO}$ & - & 0,99 & $\mathrm{~T}$ \\
\hline & $\begin{array}{l}\text { Ficus cf. enormis } \\
\text { (Mart. ex Miq.) Mart. }\end{array}$ & 2 & & $\mathrm{X}$ & & $\mathrm{NP}$ & $\mathrm{ZOO}$ & 0,95 & & $\mathrm{~T}$ \\
\hline & Ficus sp. & 1 & & $\mathrm{X}$ & & $\mathrm{NC}$ & $\mathrm{NC}$ & 0,48 & & NC \\
\hline & $\begin{array}{l}\text { Sorocea bonplandii } \\
\text { (Baill.) W.C. Burger, Lanj. } \\
\text { \& Wess. Boer }\end{array}$ & 5 & $\mathrm{X}$ & & & $\mathrm{NP}$ & $\mathrm{ZOO}$ & 4,08 & & $\mathrm{R}$ \\
\hline \multirow[t]{19}{*}{ Myrtaceae } & $\begin{array}{l}\text { Calyptranthes grandifolia } \\
\text { O. Berg }\end{array}$ & 3 & $\mathrm{X}$ & $\mathrm{X}$ & $\mathrm{X}$ & $\mathrm{P}$ & $\mathrm{ZOO}$ & 1,29 & 2,3 & $\mathrm{~T}$ \\
\hline & $\begin{array}{l}\text { Campomanesia } \\
\text { guazumifolia (Cambess.) } \\
\text { O. Berg }\end{array}$ & 2 & & & $\mathrm{X}$ & $\mathrm{NP}$ & $\mathrm{ZOO}$ & 0,48 & 1,4 & $\mathrm{~T}$ \\
\hline & $\begin{array}{l}\text { Campomanesia } \\
\text { xanthocarpa Mart. } \\
\text { ex O. Berg }\end{array}$ & 1 & $\mathrm{X}$ & & & $\mathrm{NP}$ & $\mathrm{ZOO}$ & 0,82 & & $\mathrm{~T}$ \\
\hline & Eugenia bimarginata DC. & 11 & $\mathrm{X}$ & & & NP & $\mathrm{ZOO}$ & 3,87 & & $\mathrm{R}$ \\
\hline & Eugenia florida DC. & 4 & $\mathrm{X}$ & $\mathrm{X}$ & & $\mathrm{NP}$ & $\mathrm{ZOO}$ & 1,52 & & $\mathrm{R}$ \\
\hline & Eugenia pluriflora DC. & 27 & $\mathrm{X}$ & $\mathrm{X}$ & & $\mathrm{NP}$ & $\mathrm{ZOO}$ & 8,86 & & $\mathrm{R}$ \\
\hline & $\begin{array}{l}\text { Myrcia guianensis } \\
\text { (Aubl.) DC. }\end{array}$ & 3 & $\mathrm{X}$ & $\mathrm{X}$ & & $\mathrm{NP}$ & $\mathrm{ZOO}$ & 1,6 & & $\mathrm{R}$ \\
\hline & Myrcia hebepetala DC. & 2 & & & $\mathrm{X}$ & $\mathrm{P}$ & $\mathrm{ZOO}$ & - & 2,05 & $\mathrm{~T}$ \\
\hline & $\begin{array}{l}\text { Myrcia multiflora } \\
\text { (Lam.) DC. }\end{array}$ & 1 & & & $\mathrm{X}$ & NP & $\mathrm{ZOO}$ & - & 0,97 & $\mathrm{~T}$ \\
\hline & $\begin{array}{l}\text { Myrcia selloi (Spreng.) } \\
\text { N. Silveira }\end{array}$ & 8 & & $\mathrm{X}$ & & NP & $\mathrm{ZOO}$ & 0,63 & & $\mathrm{~T}$ \\
\hline & $\begin{array}{l}\text { Myrcia splendens } \\
\text { (Sw.) DC. }\end{array}$ & 10 & $\mathrm{X}$ & $\mathrm{X}$ & $\mathrm{X}$ & NP & $\mathrm{ZOO}$ & 2,1 & 0,98 & $\mathrm{~T}$ \\
\hline & $\begin{array}{l}\text { Myrcia tomentosa } \\
\text { (Aubl.) DC. }\end{array}$ & 2 & & $\mathrm{X}$ & $\mathrm{X}$ & NP & $\mathrm{ZOO}$ & 0,48 & 1 & $\mathrm{~T}$ \\
\hline & Myrcia venulosa DC. & 6 & & $\mathrm{X}$ & & NP & $\mathrm{ZOO}$ & 2,85 & & $\mathrm{~T}$ \\
\hline & Myrcia sp1 & 1 & & & $\mathrm{X}$ & $\mathrm{NC}$ & NC & - & 0,96 & NC \\
\hline & Myrcia sp2 & 1 & & & $\mathrm{X}$ & $\mathrm{NC}$ & $\mathrm{NC}$ & - & 1,07 & NC \\
\hline & $\begin{array}{l}\text { Myrciaria cuspidate } \\
\text { O. Berg }\end{array}$ & 1 & $\mathrm{X}$ & & & NP & $\mathrm{ZOO}$ & 0,82 & & $\mathrm{R}$ \\
\hline & $\begin{array}{l}\text { Myrciaria floribunda } \\
\text { (H. West ex Willd.) O. Berg }\end{array}$ & 2 & & $\mathrm{X}$ & & NP & $\mathrm{ZOO}$ & 0,47 & & $\mathrm{~T}$ \\
\hline & $\begin{array}{l}\text { Myrciaria plinioides } \\
\text { D. Legrand }\end{array}$ & 1 & & $\mathrm{X}$ & & NP & $\mathrm{ZOO}$ & 0,48 & & $\mathrm{R}$ \\
\hline & Psidium guajava L. & 1 & & & $\mathrm{X}$ & $\mathrm{P}$ & $\mathrm{ZOO}$ & - & 1,08 & $\mathrm{~T}$ \\
\hline Nyctaginaceae & $\begin{array}{l}\text { Guapira opposita } \\
\text { (Vell.) Reitz }\end{array}$ & 4 & $\mathrm{X}$ & $\mathrm{X}$ & & NP & $\mathrm{ZOO}$ & 2,07 & & $\mathrm{R}$ \\
\hline Peraceae & $\begin{array}{l}\text { Pera glabrata (Schott) } \\
\text { Poepp. ex Baill. }\end{array}$ & 13 & & & $\mathrm{X}$ & $\mathrm{P}$ & $\mathrm{ZOO}$ & - & 16,06 & $\mathrm{~T}$ \\
\hline \multirow[t]{2}{*}{ Piperaceae } & Piper amalago L. & 16 & & $\mathrm{X}$ & & $\mathrm{P}$ & $\mathrm{ZOO}$ & 0,95 & & $\mathrm{R}$ \\
\hline & Piper arboreum Aubl. & 2 & & $\mathrm{X}$ & $\mathrm{X}$ & $\mathrm{P}$ & $\mathrm{ZOO}$ & 0,48 & 0,97 & $\mathrm{R}$ \\
\hline
\end{tabular}

Revista Árvore, Viçosa-MG, v.38, n.5, p.799-809, 2014 
Tabela 1 - Cont.

Table 1 - Cont.

\begin{tabular}{|c|c|c|c|c|c|c|c|c|c|c|}
\hline$\overline{\text { Polygalaceae }}$ & $\begin{array}{l}\text { Acanthocladus brasiliensis } \\
\text { (Klotzsch ex A. St.-Hil. } \\
\text { \& Moq.) Hassk. }\end{array}$ & 19 & $\bar{x}$ & $\mathrm{X}$ & & $\mathrm{NP}$ & NC & 4,88 & & $\mathrm{R}$ \\
\hline Primulaceae & Myrsine umbellata Mart. & 1 & & & $\mathrm{X}$ & $\mathrm{P}$ & $\mathrm{ZOO}$ & - & 1,13 & $\mathrm{~T}$ \\
\hline Proteaceae & $\begin{array}{l}\text { Roupala montana var. } \\
\text { paraensis (Huber) K.S. } \\
\text { Edwards }\end{array}$ & 2 & & & $\mathrm{X}$ & NP & ANE & - & 2,13 & $\mathrm{~T}$ \\
\hline Rosaceae & $\begin{array}{l}\text { Eriobotrya japonica } \\
\text { (Thunb.) Lindl. }\end{array}$ & 2 & & $\mathrm{X}$ & $\mathrm{X}$ & EX & EX & 0,48 & 1,01 & NC \\
\hline \multirow[t]{2}{*}{ Rubiaceae } & $\begin{array}{l}\text { Psychotria } \\
\text { carthagenensis Jacq. }\end{array}$ & 1 & & $\mathrm{X}$ & & NP & $\mathrm{ZOO}$ & 0,48 & & $\mathrm{R}$ \\
\hline & $\begin{array}{l}\text { Tocoyena formosa (Cham. } \\
\text { \& Schltdl.) K. Schum. }\end{array}$ & 1 & & & $\mathrm{X}$ & NP & $\mathrm{ZOO}$ & - & 1,11 & $\mathrm{~T}$ \\
\hline \multirow[t]{3}{*}{ Rutaceae } & Almeidea lilacina A. St.-Hil. & 2 & & $\mathrm{X}$ & & $\mathrm{P}$ & NC & 0,95 & & $\mathrm{~T}$ \\
\hline & Esenbeckia leiocarpa Engl. & 2 & $\mathrm{X}$ & $\mathrm{X}$ & & NP & AUT & 1,29 & & $\mathrm{~T}$ \\
\hline & $\begin{array}{l}\text { Zanthoxylum riedelianum } \\
\text { Engl. }\end{array}$ & 2 & & $\mathrm{X}$ & $\mathrm{X}$ & $\mathrm{P}$ & $\mathrm{ZOO}$ & 0,48 & 0,97 & $\mathrm{~T}$ \\
\hline \multirow[t]{3}{*}{ Salicaceae } & Casearia decandra Jacq. & 2 & & & $\mathrm{X}$ & NP & $\mathrm{ZOO}$ & - & 1,92 & $\mathrm{~T}$ \\
\hline & Casearia sylvestris $\mathrm{Sw}$. & 41 & & $\mathrm{X}$ & $\mathrm{X}$ & $P$ & $\mathrm{ZOO}$ & 0,63 & 22,27 & $\mathrm{~T}$ \\
\hline & Casearia obliqua Spreng. & 2 & & $\mathrm{X}$ & & NP & $\mathrm{ZOO}$ & 0,95 & & $\mathrm{~T}$ \\
\hline \multirow[t]{5}{*}{ Sapindaceae } & $\begin{array}{l}\text { Allophylus edulis (A. } \\
\text { St.-Hil. et al.) Hieron. } \\
\text { ex Niederl. }\end{array}$ & 8 & $\mathrm{X}$ & $\mathrm{X}$ & $\mathrm{X}$ & $\mathrm{P}$ & $\mathrm{ZOO}$ & 3,46 & 0,9 & $\mathrm{~T}$ \\
\hline & Cupania tenuivalvis Radlk. & 5 & & $\mathrm{X}$ & & $\mathrm{P}$ & $\mathrm{ZOO}$ & 0,79 & & $\mathrm{~T}$ \\
\hline & Cupania vernalis Cambess. & 20 & $\mathrm{X}$ & $\mathrm{X}$ & $\mathrm{X}$ & $\mathrm{P}$ & $\mathrm{ZOO}$ & 4,31 & 1,03 & $\mathrm{~T}$ \\
\hline & $\begin{array}{l}\text { Matayba elaeagnoides } \\
\text { Radlk. }\end{array}$ & 9 & & $\mathrm{X}$ & $\mathrm{X}$ & $\mathrm{P}$ & $\mathrm{ZOO}$ & 0,66 & 1,92 & $\mathrm{~T}$ \\
\hline & Sapindaceae sp1 & 1 & $\mathrm{X}$ & & & $\mathrm{NC}$ & NC & 0,82 & & NC \\
\hline Sapotaceae & $\begin{array}{l}\text { Chrysophyllum marginatum } \\
\text { (Hook. \& Arn.) Radlk. }\end{array}$ & 19 & $\mathrm{X}$ & $\mathrm{X}$ & $\mathrm{X}$ & NP & $\mathrm{ZOO}$ & 5,36 & 9,53 & $\mathrm{~T}$ \\
\hline \multirow[t]{2}{*}{ Siparunaceae } & $\begin{array}{l}\text { Siparuna brasiliensis } \\
\text { (Spreng.) A.DC. }\end{array}$ & 1 & & $\mathrm{X}$ & & $\mathrm{P}$ & $\mathrm{ZOO}$ & 0,48 & & $\mathrm{~T}$ \\
\hline & Siparuna guianensis Aubl. & 2 & & $\mathrm{X}$ & $\mathrm{X}$ & $\mathrm{P}$ & $\mathrm{ZOO}$ & 0,48 & 1 & $\mathrm{~T}$ \\
\hline Solanaceae & $\begin{array}{l}\text { Cestrum strigilatum } \\
\text { Ruiz \& Pav. }\end{array}$ & 1 & & $\mathrm{X}$ & & $\mathrm{P}$ & $\mathrm{ZOO}$ & 0,48 & & $\mathrm{~T}$ \\
\hline Tiliaceae & Tiliaceae sp1 & 1 & $\mathrm{X}$ & & & NC & NC & 0,82 & & NC \\
\hline Urticaceae & Cecropia hololeuca Miq. & 1 & & & $\mathrm{X}$ & $\mathrm{P}$ & $\mathrm{ZOO}$ & - & 1,01 & $\mathrm{~T}$ \\
\hline Verbenaceae & Lippia corymbosa Cham. & 2 & & $\mathrm{X}$ & & NP & AUT & 0,95 & & $\mathrm{R}$ \\
\hline Violaceae & Hybanthus sp. & 1 & $\mathrm{X}$ & & & NC & NC & 0,82 & & NC \\
\hline \multirow[t]{2}{*}{ Indeterminadas } & 18 morfoespécies & 22 & $\mathrm{X}$ & & & $\mathrm{NC}$ & NC & - & & - \\
\hline & 5 morfoespécies & 5 & & & $\mathrm{X}$ & $\mathrm{NC}$ & NC & - & & - \\
\hline
\end{tabular}

A riqueza estimada, utilizando-se a análise de rarefação (Figura 2), foi maior e mais similar nas classes 2 e 3. Os valores de similaridades foram baixos entre as classes em todos os índices considerados (Jaccard, Sørensen e Morisita-Horn) (Tabela 2). Os resultados da análise $\beta_{\mathrm{RC}}$ indicam ainda que todas as classes são significativamente diferentes entre si. Dois dos três pares de classes apresentam valores virtualmente máximos de significância (classes 1 e 3: 0.99 e classes 2 e 3: 1).
As famílias mais representativas na classe 1 foram Myrtaceae (8), Fabaceae (4) e Euphorbiaceae (4); na classe 2, Myrtaceae (10), Meliaceae (7) e Fabaceae (5); e na classe 3, Fabaceae (17), Myrtaceae (10) e Lauraceae (7). As espécies que apresentaram maiores valores de RN (classes 1 e 2) foram Actinostemon concepcionis, Eugenia pluriflora e Mollinedia clavigera (Figura 2). Já na classe 3 as espécies que apresentaram maiores VI foram Casearia sylvestris, 

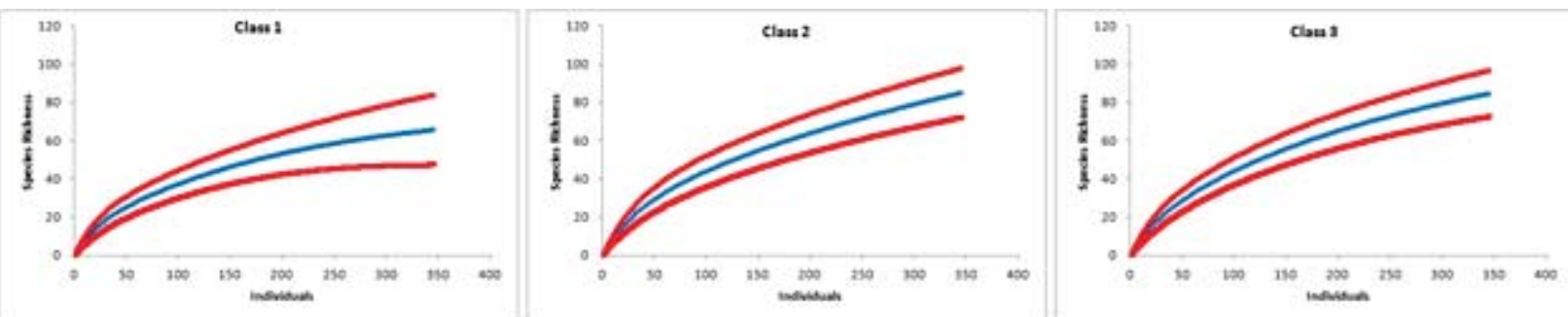

Figura 2 - Riqueza estimada entre as classes de tamanhos 1, 2 e 3 das plantas em fragmento de FES em Sorocaba, SP. A curva de rarefação extrapolada foi calculada no EstimateS, considerando 345 indivíduos. As curvas em azul representam a riqueza média e as curvas em vermelho, o intervalo de confiança de 95\% (Classes: 1, pelo menos 2 pares de folhas e $\leq 100 \mathrm{~cm}$ de altura; 2, >100 cm de altura e circunferência em nível do solo $\leq 15 \mathrm{~cm}$; e 3, circunferência à altura do peito $\geq 15 \mathrm{~cm}$ ).

Figure 2 - Estimated richness among classes 1, 2 and 3 of plants in SSF fragment in Sorocaba, SP. The extrapolated rarefaction curve was calculated in EstimateS considering 345 individuals. Blue curves represent the mean richness and the red curves represent the 95\% confidence interval (Classes: 1, at least 2 pairs of leaves and $\leq 100 \mathrm{~cm}$ of height; 2, $>100 \mathrm{~cm}$ of height and circumference at soil level $\leq 15 \mathrm{~cm}$; and 3, circumference at breast height $\geq 15 \mathrm{~cm}$ ).

Tabela 2 - Grupos sucessionais, síndromes de dispersão e índices de similaridade das três classes amostradas em remanescente de FES em Sorocaba, SP. Para similaridade gerada por modelos nulos (âRC), valores próximos a 1 representam comunidades distintas do esperado aleatoriamente e 0 , áreas similares.

Table 2 - Successional groups, dispersion syndromes and similarity indices of the three classes sampled in SSF fragment in Sorocaba, SP. For similarity generated by null models (âRC), values near 1 represent communities different from the randomly expected and 0 represents similar areas.

\begin{tabular}{|c|c|c|c|c|c|c|}
\hline Parâmetro & Classe 1 & Classe 2 & Classe 3 & Classe 1 e 2 & Classe 2 e 3 & Classe 1 e 3 \\
\hline $\mathrm{n}^{\circ}$ de ind. & 164 & 345 & 320 & - & - & - \\
\hline $\mathrm{n}^{\circ}$ de espécies & 63 & 69 & 79 & - & - & - \\
\hline ind. não identificados & $28,1 \%$ & 0 & $6,3 \%$ & - & - & - \\
\hline \% de espécies Pioneiras & 20,6 & 33,3 & 32,9 & - & - & - \\
\hline$\%$ de ind. Pioneiros & 19,4 & 33,0 & 38,1 & - & - & - \\
\hline \% de espécies Não Pioneiras & 41,3 & 58,0 & 53,2 & - & - & - \\
\hline \% de ind. Não Pioneiros & 63,5 & 64,9 & 51,9 & - & - & - \\
\hline \% de espécies Zoocóricas & 46,0 & 72,5 & 64,6 & - & - & - \\
\hline \% de ind. Zoocóricos & 64,1 & 73,2 & 72,5 & - & - & - \\
\hline \% de espécies Anemocóricas & 4,35 & 4,35 & 11,4 & - & - & - \\
\hline \% de ind. Anemocóricos & 4,71 & 0,87 & 9,69 & - & - & - \\
\hline \% de espécies Autocóricas & 8,7 & 11,6 & 10,1 & - & - & - \\
\hline \% de ind. Autocóricos & 10,0 & 19,4 & 7,81 & - & - & - \\
\hline Jaccard & - & - & - & 0,24 & 0,186 & 0,231 \\
\hline Sorensen & - & - & - & 0,388 & 0,314 & 0,375 \\
\hline Morisita-Horn & - & - & - & 0,409 & 0,177 & 0,252 \\
\hline$\beta R C$ & - & - & - & 0,76 & 1 & 0,99 \\
\hline
\end{tabular}

Lithrea molleiodes, Copaifera langsdorffii, Cryptocarya moschata, Gochnatia polymorpha, Pera glabrata, Protium heptaphyllum, Persea pyrifolia e Chrysophyllum marginatum (Figura 2). Somente Chrysophyllum marginatum e Gochnatia polymorpha estão entre as 10 espécies que apresentaram os maiores valores de RN e VI. Houve ainda espécies que foram exclusivas a uma das classes de tamanho (Tabela 1), a exemplo de Mollinedia widgrenii na classe 1,
Aspidosperma olivaceum na classe 2 e Machaerium villosum na classe 3 . Nas três classes, o grupo de não pioneiras e de dispersão zoocórica foram predominantes em número de espécies e indivíduos (Tabela 2).

Considerando a estratificação, as três classes juntas apresentaram 500 (60,4\%) indivíduos e 83 espécies (57,2\%) transitórias (típicos de dossel), bem como 268 (39,6\%) indivíduos e 28 espécies (18,4\%) residentes

Revista Árvore, Viçosa-MG, v.38, n.5, p.799-809, 2014 
(típicos de sub-bosque). Nas classes 1 e 2, 47,1\% das espécies foram consideradas transitórias e 26,9\%, residentes.

\section{DISCUSSÃO}

Considerando cada classe separadamente (Tabela 2), é possível observar que a riqueza amostrada neste estudo foi maior que a encontrada por Siminski et al. (2004), que, utilizando metodologia similar, amostraram 47 espécies no total de remanescente florestal de mesma idade, no domínio Mata Atlântica. Além disso, a riqueza de espécies do sub-bosque (classes 1 e 2) foi superior ao do dossel (classe 3 ), indicando que, mesmo que pequeno e jovem, o remanescente apresenta características similares às de fragmentos florestais maduros (TABARELLI; MANTOVANI, 1999).

A proporção de indivíduos não pioneiros deste estudo é equivalente ao encontrado em remanescente de FES considerado maduro, estudado em Campinas, SP(KINOSHITAet al., 2006), porém inferior ao encontrado em remanescente de vegetação de Floresta Ombrófila Densa com idade equivalente (60 anos) em São Pedro de Alcântara, SC (85\% de espécies não pioneiras) (SIMINSKI et al., 2004). Entre outros fatores, a diferença pode estar relacionada ao fato de o remanescente apresentar períodos de deciduidade característicos das florestas estacionais, com maior entrada de luz e favorecimento da colonização por espécies pioneiras (GANDOLFI, 2000).
Ao contrário do esperado, houve o predomínio de espécies zoocóricas nas três classes de tamanho, sendo as maiores porcentagens encontradas na classe 2 , a qual já passou pela pressão de seleção inicial (SWAINE; WITMORE, 1988). Esse mecanismo de dispersão é comumente predominante em FES em estágio sucessional avançado (KINOSHITA et al., 2006), bem como em outras fitofisionomias maduras da Mata Atlântica (LIEBSCH et al., 2008).

As famílias mais representativas neste estudo coincidem com as de outros estudos em FES no Estado de São Paulo (OLIVEIRA-FILHO; FONTES 2000; SANTOS; KINOSHITA, 2003; KINOSHITA et al., 2006), sendo Myrtaceae aquela com maior número de espécies entre as classes 1 e 2 . Esse resultado indica que o processo sucessional está avançando, pois as espécies dessa família são típicas de sub-bosque (residentes), tolerantes à sombra e zoocóricas (TABARELLI; MANTOVANI, 1999).

As 10 espécies de maior VI compreendem 53\% do valor total desse índice (Figura 3), como encontrado na maioria dos estudos desta natureza (HARTSHORN, 1980). Entre essas, cinco são pioneiras e cinco são não pioneiras (Tabela 1), indicando que o dossel está em estágio médio de regeneração (DISLICH, 2001). Em contrapartida, as espécies com maiores valores de regeneração natural (RN) (Figura 3) são consideradas como não pioneiras e residentes (típicas de sub-bosque), sugerindo que o remanescente está proporcionando
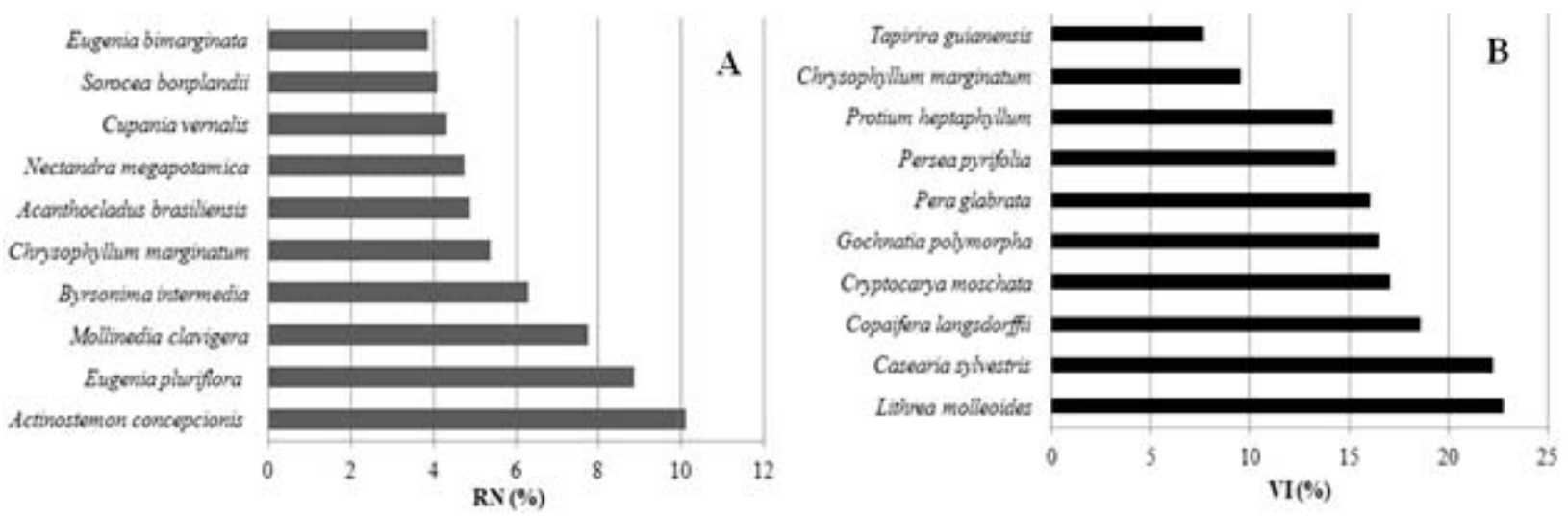

Figura 3 - Regeneração natural (RN) e valor de importância (VI) das espécies amostradas em remanescente de Floresta Estacional Semidecidual em Sorocaba, SP.

Figure 3 - Natural Regeneration (NR) and Importance Value (IV) of the species sampled in the fragment of Semideciduous Seasonal Forest in Sorocaba, SP. 
condições microclimáticas para entrada e permanência de espécies que necessitam de áreas mais sombreadas. Além disso, Aspidosperma olivaceum, Cordia trichotoma, Ocotea elegans, Guarea guidonia, Psychotria carthagenensis, Esenbeckia leiocarpa e Casearia obliqua, que são espécies não pioneiras e transitórias (típicas de dossel), foram amostradas somente nas classes 1 e 2 .

Os aspectos relacionados vão contra a hipótese inicial deste trabalho, e essas características, aliadas à baixa similaridade encontrada entre as classes, indicam que a diferença de composição florística e a estrutura permitirão que, no futuro, ocorram a substituição e enriquecimento de espécies no dossel e, consequentemente, avanço sucessional.

\section{CONCLUSÃO}

O remanescente de Floresta Estacional Semidecidual estudado encontra-se em estágio intermediário de sucessão, apresenta regeneração com elevada riqueza de espécies transitórias, formada em sua maioria por espécies não pioneiras e zoocóricas. Esse fragmento possibilita a entrada e estabelecimento de significativa diversidade de espécies residentes (típicas de subbosque) e zoocóricas, em detrimento das espécies heliófitas e pioneiras. Dessa maneira, a hipótese inicial não foi corroborada, pois ainda que o remanescente seja pequeno e relativamente jovem, com histórico de intensa degradação, abriga alta diversidade de espécies, com atributos que permitem a continuidade do avanço sucessional e a manutenção dessa diversidade.

\section{AGRADECIMENTOS}

Ao CNPq, pela concessão das bolsas de Iniciação Científica; à Capes, pela concessão da Bolsa de Mestrado; aos Professores Jorge Y. Tamashiro e Fiorella Manzini, Leonardo Meirelles e Thiago Mouzinho, pelo auxílio na identificação do material coletado; aos curadores dos Herbários ESA, UEC, SPSF e SP, pelo suporte; e aos técnicos Almir Calazans da Silva, Ivonir Piotrowski Santos e Cícero Santos Branco, pelo auxílio no trabalho de campo.

\section{REFERÊNCIAS}

AIZEN, M.A.; FEINSINGER, P. Habitat fragmentation, native insect pollinators, and feral honey bees in Argentine (Chaco Serrano). Ecological Applications.v.4, n.2, p.378-392, 1994.
APG III. An update of the Angiosperm Phylogeny Group classification for the orders and families of flowering plants: APG III. Botanical Journal of the Linnean Society, v.161, n.2, p.105-121, 2009.

CARDOSO-LEITE, E.; RODRIGUES, R. R.

Fitossociologia e caracterização sucessional de um fragmento de Floresta Estacional no Sudeste do Brasil. Revista Árvore, Viçosa, MG,v.32, n.3, p.583-595, 2008.

CHAO, A. Species richness estimation. In: BALAKRISHNAN, N.; READ, C.; VIDAKOVIC, B. (Ed.). Encyclopedia of Statistical Sciences. New York: Wiley, 2005. p.7909-7916

CHASE, J. M. et al. Using null models to disentangle variation in community dissimilarity from variation in á-diversity. Ecosphere, v.2, n.2, p.1-11, 2011.

COLWELL, R. K. EstimateS: Statistical estimation of species richness and shared species from samples. Disponível em: <http://purl.oclc.org/ estimates>. Acesso em: 07 mar. 2014.

DISLICH, R.; CERSÓSIMO, L.; MANTOVANI, W. Análise da estrutura de fragmentos florestais no Planalto Paulistano-SP. Revista Brasileira de Botânica, v.24, n.3, p.321-332, 2001.

ENGEL, V. L.; PARROTA, J. A. Definindo a restauração ecológica: tendências e perspectivas mundiais. In: KAGEYAMA, P. Y. et al. (Org.). Restauração ecológica de ecossistemas naturais. Botucatu: FEPAF, 2008. p.49-76

FENNER, M. Seedlings. The New

Phytologist, v.106, n.1, p.35-47, 1987.

GANDOLFI, S. História natural de uma floresta estacional Semidecidual no município de Campinas, São Paulo, Brasil. 2000. 520f. Tese (Doutorado em Biologia Vegetal) - Instituto de Biologia, Universidade Estadual de Campinas, Campinas, 2000.

GILLIAM, F. S. et al. Ecosystem processes: herbaceous layer and soil response to experimental acidification in a central appalachian hardwood forest. Journal of Environmental

Quality, v.23, p.835-844, 1994. 
HARTSHORN, G. S. Neotropical forests dynamics. Biotropica, v.12, n.1, p.23-30, 1980.

INSTITUTO BRASILEIRO DE GEOGRAFIA E ESTATÍSTICA - IBGE. Manual técnico da vegetação brasileira. Rio de Janeiro, 2012. 271p.

KINOSHITA, L. S. et al. Composição florística e síndromes de polinização e de dispersão da mata do Sítio São Francisco, Campinas, SP, Brasil. Acta Botanica Brasilica, v.20, n.2, p.313-327, 2006.

KÖEPPEN, W. Climatologia: com um estudio de los climas de la tierra. México: Fundo de Cultura Econômica, 1948.

KRONKA, F. J. N. et al. Inventário Florestal da Vegetação Natural do Estado de São Paulo. São Paulo: Secretaria do Meio Ambiente, Instituto Florestal; Imprensa Oficial, 2005. 200p.

LIEBSCH, D.; MARQUES, M. C. M.; GOLDENBERG, R. How long does the Atlantic Rain Forest take to recover after a disturbance? Changes in species composition and ecological features during secondary succession.

Biological Conservation, v.141, p.17171725, 2008.

\section{LISTA DE ESPÉCIES DA FLORA DO BRASIL.} Lista de Espécies da Flora do Brasil. 2014. Disponível em: <http://

floradobrasil.jbrj.gov.br/>. Acesso em: 05 Jan. 2014.

LOPES, S. F. et al. An ecological comparison of floristic composition in seasonal semideciduous forest in southeast Brazil: implications for conservation. International Journal of Forestry Research, v.2012, p.1-14, 2012.

MAGURRAN, A. E. Measuring biological diversity. Oxford: Blackwell Publishing, 2004. 256p.

MÜELLER-DOMBOIS, D.; ELLENBERG, H. Aims and methods of vegetation ecology. New York: Wiley, 1974. 547p.

OKSANEN, F. et al. Vegan: community ecology package. Disponível em: <http://cran.r-project.org/ web/packages/vegan/vegan.pdf>. Acesso em: 07 mar. 2014.
OLIVEIRA, J. B. Solos do Estado de São Paulo: descrição das classes registradas no mapa pedológico. Campinas: Instituto Agronômico de Campinas, 1999. 122p.

OLIVEIRA-FILHO, A. T.; FONTES, M. A. Patterns of floristic differentiation among Atlantic Forests in Southeastern Brazil and the influence of climate. Biotropica, v.32, p.793-810, 2000.

R CORE TEAM. R: A Language and Environmental for Statistical Computing. Vienna: 2014. Disponível em:<http://www.r-project.org>. Acesso em: 07 mar. 2014.

RIBEIRO, M. C. et al. The brazilian atlantic forest: how much is left, and how is the remaining forest distributed? Implications for conservation. Biological Conservation, v.142, p.1141-1153, 2009.

SANTOS, K.; KINOSHITA, L. S. Flora arbustivoarbórea do fragmento de floresta estacional semidecidual do ribeirão cachoeira, município de Campinas, SP. Acta Botanica Brasilica, v.17, n.3, p.325-341, 2003.

SHEPHERD, G. J. FITOPAC 2.1 (versão preliminar). Campinas: Departamento de Biologia Vegetal, Universidade Estadual de Campinas, 2009.

SWAINE, M. D.; WHITMORE, T. C. On the definition of ecological species groups in tropical forests. Vegetatio, v.75, p.81-86, 1988.

SIMINSKI, A. et al. Sucessão florestal secundária no município de São Pedro de Alcântara, litoral de Santa Catarina: estrutura e diversidade. Ciência Florestal, v.14, n.1, p.21-33, 2004.

TABARELLI, M.; MANTOVANI, W. A regeneração de uma floresta tropical montana, após corte e queima, São Paulo, Brasil. Revista Brasileira de Biologia, v.59, n.2, p.239-250, 1999.

VOLPATO, M. M. L. Regeneração natural em uma floresta secundária no domínio de Mata Atlântica: uma análise fitossociológica. 1994. 123f. Dissertação (Mestrado em Ciência Florestal) Universidade Federal de Viçosa, Viçosa, MG, 1994.

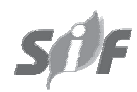

Revista Árvore, Viçosa-MG, v.38, n.5, p.799-809, 2014 